\title{
Deep-core photoionization of krypton atoms below and above the 1s ionization threshold
}

\author{
N. Boudjemia $\odot,{ }^{1, *}$ K. Jänkälä $\odot,{ }^{1}$ R. Püttner $\odot,{ }^{2}$ T. Gejo, ${ }^{3,4}$ L. Journel,,${ }^{3,5}$ Y. Kohmura, ${ }^{3}$ \\ M. Huttula, ${ }^{1}$ M. N. Piancastelli ${ }^{1},{ }^{3,5,6}$ M. Simon, ${ }^{3,5}$ and M. Oura $\oplus^{3}$ \\ ${ }^{1}$ Nano and Molecular Systems Research Unit, University of Oulu, P.O. Box 3000, 90014 Oulu, Finland \\ ${ }^{2}$ Fachbereich Physik, Freie Universität Berlin, Arnimallee 14, D-14195 Berlin, Germany \\ ${ }^{3}$ RIKEN SPring-8 Center, 1-1-1 Kouto, Sayo-cho, Sayo-gun, Hyogo 679-5148, Japan \\ ${ }^{4}$ Graduate School of Materials Science, University of Hyogo, Kamigori-cho, Ako-gun, Hyogo 678-1297, Japan \\ ${ }^{5}$ Sorbonne Université, CNRS, Laboratoire de Chimie Physique-Matière et Rayonnement, LCPMR, F-75005 Paris, France \\ ${ }^{6}$ Department of Physics and Astronomy, Uppsala University, SE-75120 Uppsala, Sweden
}

(Received 20 January 2020; accepted 6 April 2020; published 4 May 2020)

\begin{abstract}
Electronic relaxation of atomic Kr below and above the $1 s$ ionization threshold is investigated experimentally using hard x-ray photoelectron spectroscopy. The experimental results are interpreted with the aid of relativistic Dirac-Fock calculations. The $1 s$ orbital core-hole lifetime is extracted and the satellite's structures accompanying the photoelectron main line are assigned. Auger spectra recorded below and above the $K$ edge are also investigated. In particular, the Auger cascade originating from vacancies of different origin in the $L$ shell is analyzed in great detail. Competition between radiative $K L$ versus nonradiative $K L L$ Auger emission is emphasized.
\end{abstract}

DOI: 10.1103/PhysRevA.101.053405

\section{INTRODUCTION}

The availability of high-resolution hard x-ray radiation up to $51 \mathrm{keV}$ at SPring-8 [1] in Japan has opened new avenues for the study of heavy atoms and molecules with hard x-ray photoelectron spectroscopy (HAXPES), which is a powerful technique for the investigation of deep-core orbitals of different materials in a gas phase [1] or condensed matter [2]. In heavy atoms, either isolated or embedded in a molecule, this new possibility to create a very deep-core hole via singlephoton excitation or ionization enables direct studies of a multitude of interesting phenomena, such as core-hole lifetime in the attosecond range $[3,4]$, direct observation of the relative weight of $K L$ emission processes versus Auger relaxation [3], and partial charge redistribution within a molecule [4].

The photoionization dynamics of $\mathrm{Kr}$ atoms have been under intense investigation in the past. Studies have been conducted in the soft $\mathrm{x}$-ray photon-energy range, which has enabled the investigation of valence photoionization, Auger decay spectra, and the formation of multiply charged $\mathrm{Kr}$ ions as a function of the photon energy above the $2 p_{3 / 2}$ ionization threshold [5-8]. Several studies using lasers have been conducted, such as measurement of x-ray transitions of $\mathrm{Kr}$ clusters [9], and pump-probe studies to trace the $M$-shell decay dynamics [10] and excitation using the OMEGA laser at the Laboratory for Laser Energetics to study $\mathrm{Kr} K$-shell $\mathrm{X}$-ray emission [11]. Kr has also been studied in very strong photon fields at free electron lasers where the focus was put to multiphoton ionization [12] and with synchrotron-based timeresolved $\mathrm{x}$-ray microprobe experiments for the investigation

\footnotetext{
*nacer.boudjemia@oulu.fi
}

of high-field orbital alignment [13]. However, HAXPES studies on $\mathrm{Kr}$ atoms following $K$-edge ionization are scarce. High photon energy has been used to investigate nondipole effects in $1 s$ photoionization [14] and X-ray absorption spectroscopy (XAS) measurements have been performed for structural determination of the hydrophobic hydration shell of $\mathrm{Kr}$ [15]. $L_{2,3} M M$ and $M_{4,5} N N$ Auger spectra have been reported using electron-impact ionization [16]. In addition, the calculated energy diagram for $\mathrm{Kr}-\mathrm{Kr}^{4+}$ states, showing the most prominent relaxation channels for neutral $\mathrm{Kr}$ and $\mathrm{Kr}$ ions, has been reported by Oura et al. [1]. The $\mathrm{Kr} K$ and the subsequent $L M X$ Auger spectra have been studied by Kovalík [17]. In this study, the initial state is induced by electron capture of the ${ }^{83} \mathrm{Rb}$ nucleus, leading to a ${ }^{83} \mathrm{Kr}$ atom with a hole in the $1 s$ shell.

The present paper provides the results of measurements carried out for $\mathrm{Kr} 1 s$ ionization and the subsequent $L X X(X=$ $M, N)$ Auger decay cascade in the electron kinetic-energy range of $1.0-1.8 \mathrm{keV}$. The present $L X X$ Auger spectra, in principle, agree with those of Kovalík [17]; however, they are measured using an experimental resolution, which is smaller by more than a factor of 10 . The Auger $L X X$ spectrum was also studied below the $K$ edge. The observed spectral features for both photoelectron and Auger electron spectra are assigned with the aid of relativistic Dirac-Fock calculations, which show good agreement with the experimental observations. The results obtained from the analysis of the photoelectron spectrum of $\mathrm{Kr}$ include the binding energy and the lifetime of the $1 s$ ionized state as well as its radiative versus nonradiative decay branching ratio. For the Auger decay, we provide a detailed analysis of the spectra starting from $2 p^{-1}$ initial states and including three decay steps going from $\mathrm{Kr}^{1+}$ up to $\mathrm{Kr}^{4+}$. 


\section{EXPERIMENT}

Experiments were carried out at the BL29XU undulator beam line of SPring-8 [18]. The $\mathrm{Kr} 1 s$ photoelectron spectrum was recorded using $19992 \mathrm{eV}$ photon energy, and the $L$-shell Auger spectrum was measured below and above the $K$ edge at about 13.0 and $16.5 \mathrm{keV}$ photon energies. The spectra were measured using a Scienta Omicron SES2002 hemispherical deflection energy analyzer with a Scienta Gc50 gas cell [19]. The target pressure outside the gas cell was about $4.5 \times 10^{-5}$ mbar during the measurements.

Energy resolution of the electron energy analyzer was $360 \mathrm{meV}$ with a pass energy of $200 \mathrm{eV}$. The kinetic-energy scale of the spectrometer was calibrated using the Ne $K L L$, $\operatorname{Ar} K L L$, and $\mathrm{Kr} L M M$ Auger spectra [20]. The photon-energy scale was calibrated via the $1 s$ binding energy of $\mathrm{Ar}, \mathrm{Xe} 1 s$, $2 p$, and $\mathrm{Kr} 1 s, 2 p$ [21]. The uncertainty of the incident photon energy has been estimated to be $3.1 \mathrm{eV}$.

The photon bandwidth was estimated to be $2.29 \pm 0.17 \mathrm{eV}$ at photon energy of $19992 \mathrm{eV}$. Determining the bandwidth of a $20 \mathrm{keV}$ photon beam is not a straightforward task due to the fact that photoelectron lines, whose natural width is narrow enough, lay at too high kinetic energies to be seen by any standard electron spectrometer.

Because of this, a different approach was applied. The Ar $1 s, \mathrm{Kr} 2 p, \mathrm{Kr} 1 s$, and $\mathrm{Xe} 1 s$ photoelectron lines were measured over a photon-energy range of 6 to $35.5 \mathrm{keV}$. These spectra were fitted with Voigt profiles using Lorentzian and Gaussian widths as free parameters. The Lorentzian widths turned out to be in reasonable agreement with theoretical values [22,23] for $\mathrm{Kr}$ and $\mathrm{Xe}$, and experimental values for $\mathrm{Ar}$ [24]. Detector broadening was then subtracted from the Gaussian component and the remaining width was compared against the broadening predicted by ray tracing simulations of the beam line. It turned out that the simulated values can be described reasonably well using a linear relation with a slope of $0.87 \pm 0.05$. One can then correct the value from the ray tracing simulation $(2.90 \mathrm{eV})$ with the obtained slope, giving a value of $2.29 \pm$ $0.17 \mathrm{eV}$ for the photon bandwidth.

\section{CAlCulations}

The calculations were carried out using the Flexible Atomic Code (FAC) [25], which uses a relativistic Dirac-FockSlater multiconfiguration atomic structure framework [26]. In this method, atomic state functions (ASFs) with the same total angular momentum, projection, and parity are constructed as linear combinations of configuration state functions (CSFs) in the jj-coupling scheme. CSFs are thus antisymmetrized linear combinations of $N$-electron Slater determinants. The one-electron wave functions in the determinants were solved in the average energy-level scheme via the self-consistent field Dirac-Fock method [26]. Then the mixing coefficients for ASFs were solved by diagonalizing the total relativistic Hamiltonian in the CSF basis. In addition to the relativistic effects provided by the Hamiltonian, Breit interaction and quantum electrodynamics corrections such as self-energy and vacuum polarization were included systematically via perturbation theory. These additional corrections are critical for the correct prediction of $1 s$ binding energies in heavy atoms [27].

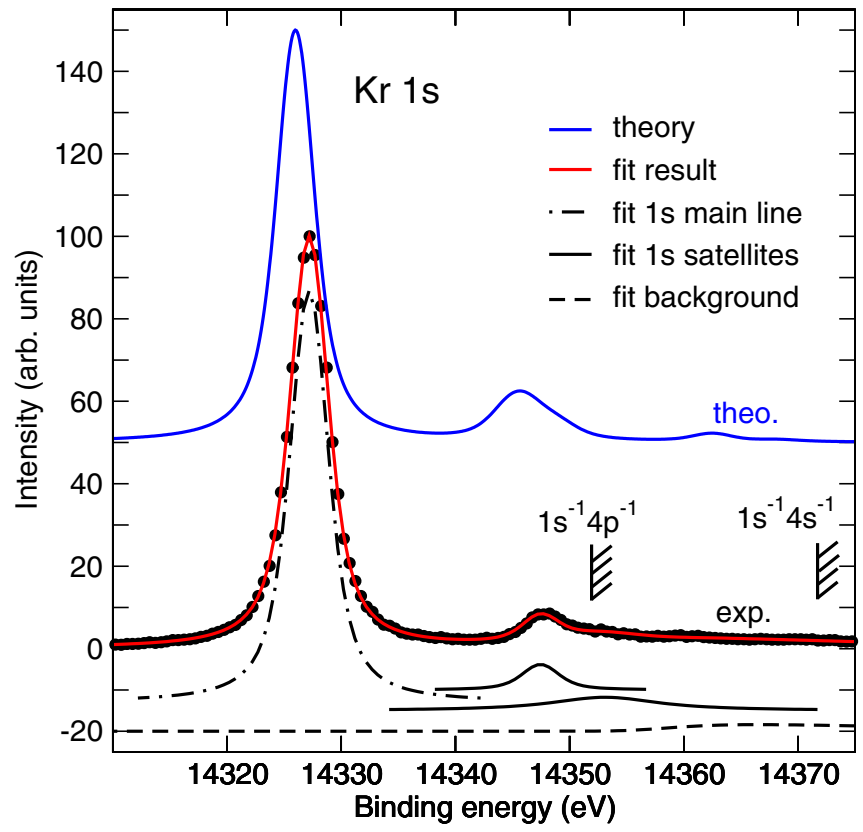

FIG. 1. Experimental and theoretical $\mathrm{Kr} 1 s$ photoelectron spectra. The $1 s^{-1} 4 p^{-1}$ and $1 s^{-1} 4 s^{-1}$ double-ionization thresholds are indicated by vertical lines.

The calculations of the singly ionized states included the $1 s^{-1}, 2 s^{-1}$, and $2 p^{-1}$ states for the main lines, as well as the $1 s^{-1} 4 s^{-1} n l$ and $1 s^{-1} 4 p^{-1} n l \quad(n l=5 s / p, 6 s / p$, and $7 s / p$ ) configurations for the satellite lines. Auger transitions in the kinetic-energy range of $1.0-1.8 \mathrm{keV}$ were calculated by including the $(2 s 2 p)^{-1}$ and $(2 s 2 p)^{-2}$ initial states in ions with charge up to $\mathrm{Kr}^{4+}$. The calculations at each Auger step included all relevant energetically allowed final states that can be constructed from the occupied orbitals, meaning $M X$ doubly, $M X X, L M X$, and $L L X$ triply, and $X X X X$ quadruply ionized states, where $X$ stands for holes in the $M$ or $N$ shell. Note that the decay of these holes does not produce electrons in the kinetic-energy region under discussion, but is needed to predict the widths and intensities of the observed lines correctly. Radiative transition rates were calculated in dipole approximation using the length gauge. Auger decay rates were calculated in the standard formulation, except for construction of continuum waves that were approximated near the nucleus by the shape of highly excited bound orbitals [25]. The cross section for $1 s$ photoionization and its satellite structure was taken from Ref. [28].

\section{RESULTS AND DISCUSSION}

\section{A. Krypton $1 s$ photoelectron spectrum}

Figure 1 shows the experimental $1 s$ photoelectron spectrum of $\mathrm{Kr}$ measured at a photon energy of $19992 \pm$ $3.1 \mathrm{eV}$. The $1 s$ binding energy extracted from the spectrum is $14327 \pm 3.2 \mathrm{eV}$, which is in agreement with the previous literature values of $14327.26 \pm 0.4$ and $14327.2 \pm 0.8 \mathrm{eV}$ $[29,30]$. The value is also in good agreement with the present FAC calculations, which give the result of $14325.49 \mathrm{eV}$, and the previous calculation of $14326.10 \mathrm{eV}$ obtained using the GRASP2K code [27]. 
TABLE I. Assignment and energy ranges of main satellite configurations. For each configuration, the lowest and highest energy is given.

\begin{tabular}{|c|c|c|c|c|}
\hline & \multicolumn{4}{|c|}{ Energy ranges of satellites spectra in $\mathrm{eV}$} \\
\hline & \multicolumn{2}{|c|}{ Distance from $1 s^{-1}$ threshold } & \multicolumn{2}{|c|}{ Binding energy } \\
\hline & $\begin{array}{l}\text { Lowest } \\
\text { value }\end{array}$ & $\begin{array}{l}\text { Highest } \\
\text { value }\end{array}$ & $\begin{array}{l}\text { Lowest } \\
\text { value }\end{array}$ & $\begin{array}{l}\text { Highest } \\
\text { value }\end{array}$ \\
\hline $4 p \rightarrow 5 p$ & 18.61 & 21.23 & 14344.04 & 14346.66 \\
\hline $4 p \rightarrow 6 p$ & 22.58 & 24.04 & 14348.24 & 14349.70 \\
\hline $4 p \rightarrow 7 p$ & 24.14 & 25.39 & 14349.92 & 14351.17 \\
\hline $4 p^{-1}$ & 24.26 & 25.34 & 14351.52 & 14352.61 \\
\hline $4 s \rightarrow 5 s$ & 36.57 & 37.73 & 14361.84 & 14363.00 \\
\hline $4 s \rightarrow 6 s$ & 42.08 & 42.97 & 14367.42 & 14368.32 \\
\hline $4 s \rightarrow 7 s$ & 44.12 & 44.98 & 14369.56 & 14370.41 \\
\hline $4 s^{-1}$ & 44.05 & 44.90 & 14371.32 & 14372.16 \\
\hline
\end{tabular}

The photoelectron spectrum in Fig. 1 was fitted using four Voigt functions: one for the main line and three for the satellite structure. The energy positions of the satellite's structures relative to the main line have been derived from the $2 s$ photoelectron spectrum (not shown here). For the $1 s$ main line, the fit provides a full width at half maximum (FWHM) of $\sim 4 \mathrm{eV}$.

The Lorentzian width of $2.65 \pm 0.14 \mathrm{eV}$ corresponds to $248 \pm 12$ as for the lifetime of the $1 s$ ionized state of atomic $\mathrm{Kr}$. The value is in agreement with the values obtained for $\mathrm{Kr}$

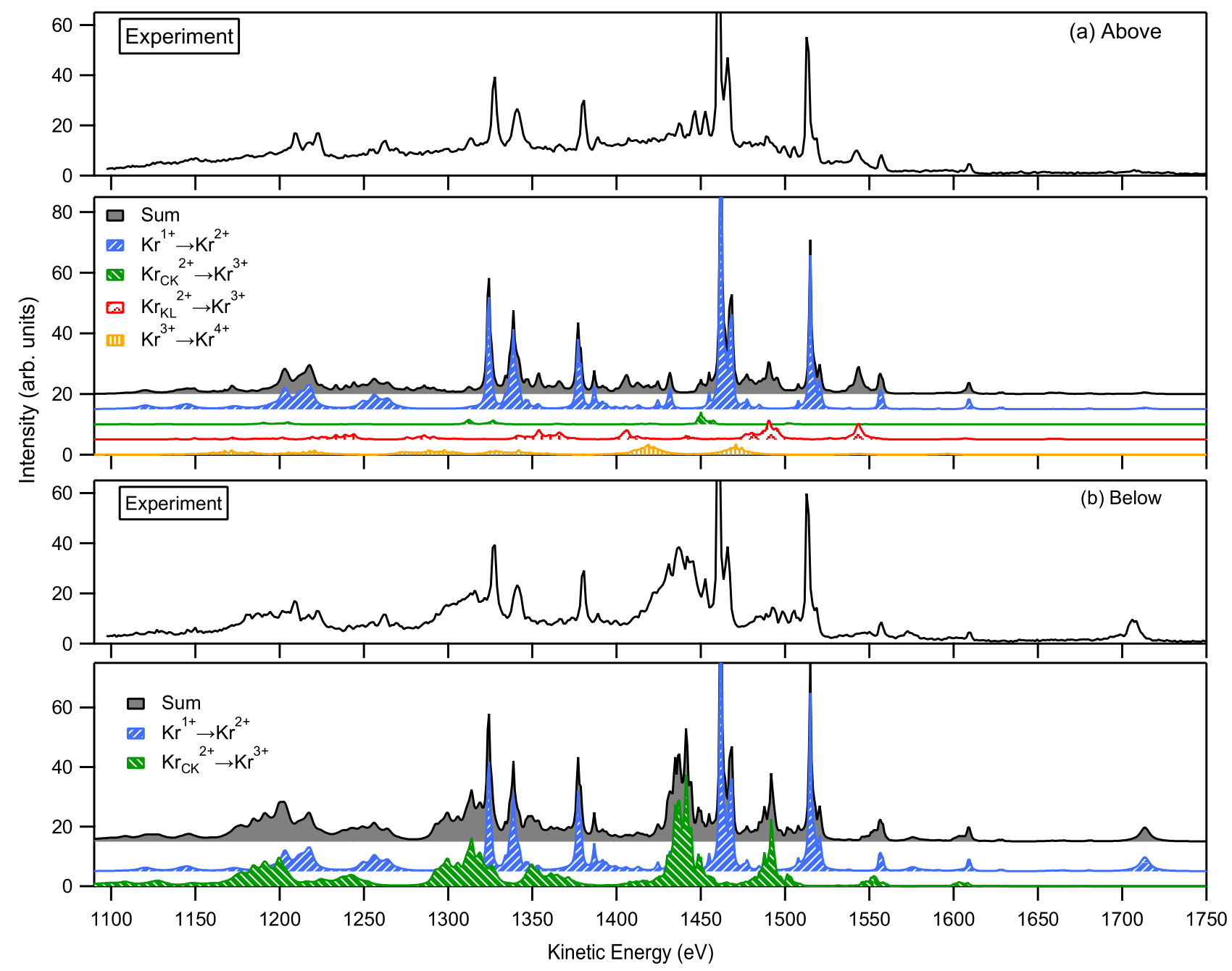

FIG. 2. Experimental and calculated Auger electron spectra (a) above and (b) below the $1 s$ ionization threshold of Kr at photon energies of 16.5 and $13.0 \mathrm{keV}$. The spectra are normalized to the peak at $1460 \mathrm{eV}$ having height of $100 . \mathrm{Kr}_{C K}^{2+}$ and $\mathrm{Kr}_{K L}^{2+}$ denote dicationic $\mathrm{Kr}$ states populated by $L L X$ Coster-Kronig Auger decay and $K L X(X=L, M, N)$ Auger decay, respectively. 
in aqueous solution (2.7 eV) [15] and from solid $\mathrm{Kr}(2.75 \mathrm{eV})$ [23].

Energy positions relative to the main line of the peak profiles fitted to the satellite structures were taken from the $2 s$ photoelectron spectrum of $\mathrm{Kr}$ (not shown here) because the spectrum can be measured with higher resolution. In the $1 s$ case, the satellite spectrum has two distinct, but, due to the lifetime broadening, rather featureless regions that are approximately 20 and $35 \mathrm{eV}$ from the main line. The first one is identified to transitions to $1 s 4 p^{-1} n l$ final states and the second to $1 s 4 s^{-1} n l$ final states. Table I provides the theoretical energy ranges for the $1 s(4 s, p)^{-1} n s, p$ satellite configurations. In general, the present calculations for the energies combined with theoretical cross sections from [28] provide qualitative agreement with the measured $1 s$ photoelectron spectrum.

\section{B. Auger decay of $\mathrm{Kr}$ below and above $1 s$ ionization threshold}

Figure 2 shows the experimental and simulated Auger decay spectra in the electron kinetic-energy range of 1075$1750 \mathrm{eV}$ measured above [Fig. 2(a)] and below [Fig. 2(b)] the $1 s$ ionization threshold. In this energy range, only Auger decays of states with at least one $L$ hole contribute to the spectrum. The fundamental difference between the two spectra recorded below and above threshold is that the population of initial states is caused by different processes, as will be discussed below. As a result, below threshold, the initial states have charge states of $\mathrm{Kr}^{1+}$ and $\mathrm{Kr}^{2+}$, and exactly one hole in the $L$ shell. Above threshold, on the other hand, the initial states range from $\mathrm{Kr}^{1+}$ to $\mathrm{Kr}^{3+}$, with a maximum of two holes in the $L$ shell.

For drawing the spectra in Fig. 2, natural widths have been included for every line individually and the total spectrum was convoluted with the experimental broadening of $360 \mathrm{meV}$. For $\mathrm{Kr}^{+} \rightarrow \mathrm{Kr}^{2+}$ and $\mathrm{Kr}_{C K}^{2+} \rightarrow \mathrm{Kr}^{3+}$ spectra, both the initialand final-state broadening were included to the natural width. For the sake of visibility, for $\mathrm{Kr}_{K L}^{2+} \rightarrow \mathrm{Kr}^{3+}$ and $\mathrm{Kr}^{3+} \rightarrow$ $\mathrm{Kr}^{4+}$ spectra, only the initial-state broadening is taken into account. It is also expected that in those cases, the initial-state broadening dominates over the final-state broadening.

Below the $1 s$ threshold, the initial states are produced solely by direct photoionization of the $L_{1,2,3}$ shells,

$$
\gamma+\mathrm{Kr} \stackrel{100 \%}{\longrightarrow} \mathrm{Kr}^{1+}(2 s 2 p)^{-1}+e_{\mathrm{ph}}
$$

In contrast, above the $1 s$ threshold, the initial states with holes in the $L$ shell are predominantly populated via $K L_{2,3}$ X-ray emission, and $K L L$ and $K L X$ Auger decay, with $X=$ $M, N$. In detail, for a photon energy of $16.5 \mathrm{keV}$, we find

$$
\begin{aligned}
\gamma+\mathrm{Kr} & \stackrel{\sim 87 \%}{\longrightarrow} e_{\mathrm{ph}}+\mathrm{Kr}^{1+}\left(1 s^{-1}\right) \\
& \stackrel{54 \%}{\longrightarrow} \mathrm{Kr}^{1+}\left(2 p^{-1}\right)+\gamma_{K \alpha} \\
& \stackrel{25 \%}{\longrightarrow} \mathrm{Kr}^{2+}(2 s 2 p)^{-2}+e_{K L L} \\
& \stackrel{8 \%}{\longrightarrow} \mathrm{Kr}^{2+}\left[(2 s 2 p)^{-1} n l^{-1}\right]+e_{K L M} .
\end{aligned}
$$

The decay probabilities for $K L$-fluorescence emission,

\begin{tabular}{|c|c|c|c|}
\hline Transition & $\begin{array}{l}\text { Initial } \\
\text { config. }\end{array}$ & $\begin{array}{c}\text { Final } \\
\text { config. }\end{array}$ & $\begin{array}{c}\text { Kinetic } \\
\text { energy }(\mathrm{eV})\end{array}$ \\
\hline $\mathrm{Kr}^{1+} \rightarrow \mathrm{Kr}^{2+}$ & $(2 s 2 p)^{-1}$ & $\begin{array}{c}3 p^{4} \\
3 d^{8} \\
3 s^{1} n l^{-1} \\
3 p^{5} n l^{-1} \\
3 d^{9} n l^{-1}\end{array}$ & $\begin{array}{c}1129-1494 \\
1430-1744 \\
1226-1711 \\
1226-1691 \\
1514-1635\end{array}$ \\
\hline $\mathrm{Kr}_{C K}^{2+} \stackrel{\text { above }}{\longrightarrow} \mathrm{Kr}^{3+}$ & $2 p^{5} 4 p^{5}$ & $\begin{array}{c}3 s^{1} 3 p^{5} 4 p^{5} \\
3 p^{4} 4 p^{5} \\
3 s^{1} 3 d^{9} 4 p^{5} \\
3 p^{5} 3 d^{9} 4 p^{5} \\
3 d^{8} 4 p^{5}\end{array}$ & $\begin{array}{c}1099-1139 \\
1175-1207 \\
1238-1258 \\
1302-1344 \\
1433-1460\end{array}$ \\
\hline $\mathrm{Kr}_{C K}^{2+} \stackrel{\text { below }}{\longrightarrow} \mathrm{Kr}^{3+}$ & $2 p^{5} n l^{-1}$ & $\begin{array}{c}3 d^{7} \\
3 d^{8} n l^{-1} \\
3 s^{1} 3 p^{5} n l^{-1} \\
3 p^{4} n l^{-1} \\
3 p^{5} 3 d^{9} n l^{-1}\end{array}$ & $\begin{array}{c}1400-1514 \\
1425-1634 \\
1073-1365 \\
1077-1442 \\
1291-1571\end{array}$ \\
\hline $\mathrm{Kr}_{L L}^{2+} \rightarrow \mathrm{Kr}^{3+}$ & $2 p^{4}$ & $\begin{array}{c}2 p^{5} 3 p^{4} \\
2 p^{5} 3 d^{8} \\
2 p^{5} 3 s^{1} n l^{-1} \\
2 p^{5} 3 p^{5} n l^{-1} \\
2 p^{5} 3 d^{9} n l^{-1}\end{array}$ & $\begin{array}{c}1140-1343 \\
1409-1614 \\
1090-1486 \\
1260-1570 \\
1527-1715\end{array}$ \\
\hline $\mathrm{Kr}_{L X}^{2+} \rightarrow \mathrm{Kr}^{3+}$ & $2 p^{5} n l^{-1}$ & $\begin{array}{c}3 p^{3} \\
3 d^{7} \\
3 p^{4} 3 d^{9} \\
3 p^{4} 4 s^{1} \\
3 p^{4} 3 p^{5} \\
3 d^{8} 4 s^{1} \\
3 d^{8} 4 p^{5}\end{array}$ & $\begin{array}{c}1127-1220 \\
1409-1517 \\
1130-1600 \\
1158-1417 \\
1164-1443 \\
1418-1511 \\
1423-1610\end{array}$ \\
\hline $\mathrm{Kr}^{3+} \rightarrow \mathrm{Kr}^{4+}$ & $2 p^{5} n l^{-2}$ & $\begin{array}{c}3 p^{3} n l^{-1} \\
3 p^{4} 3 d^{9} n l^{-1} \\
3 p^{4} n l^{-2} \\
3 p^{5} 3 d^{8} n l^{-1} \\
3 p^{5} n l^{-3} \\
3 d^{7} n l^{-1} \\
3 d^{8} n l^{-2} \\
n l^{-4} \\
3 s^{1} 3 d^{8} n l^{-1}\end{array}$ & $\begin{array}{l}1128-1510 \\
1230-1378 \\
1122-1527 \\
1366-1505 \\
1232-1384 \\
1377-1505 \\
1491-1633 \\
1382-1725 \\
1295-1416\end{array}$ \\
\hline
\end{tabular}
and the $K L L$ and $K L X$ Auger are derived from the present
TABLE II. Electronic configurations of initial and final states of $\mathrm{Kr}$ Auger decay measured below and above the 1s ionization threshold ( $n=3$ or 4$)$.

calculations and yield $54 \%, 25 \%$, and $8 \%$, respectively. The $1 s$ ionization cross section at $16.5 \mathrm{keV}$ photon energy is 11,48 , and 27 times larger than the $2 s, 2 p_{1 / 2}$, and $2 p_{3 / 2}$ ionization cross sections, respectively, leading to $8 \%$ of direct $2 s$ and $5 \%$ of direct $2 p$ ionization. Therefore, the contribution of direct $L$-shell ionization can be neglected above the $1 s$ threshold.

To understand the features of the spectra shown in Fig. 2, it is important to realize that the individual initial vacancies relax via different Auger cascades that produce overlapping features in the studied region. In the present case, we included Auger steps up to quadruply ionized $\mathrm{Kr}$, as will be discussed in detail in the following sections. Table II provides an electron configuration level summary of the possible Auger decay paths and their calculated kinetic-energy ranges. In the following, we shall discuss each initial decay step individually. 


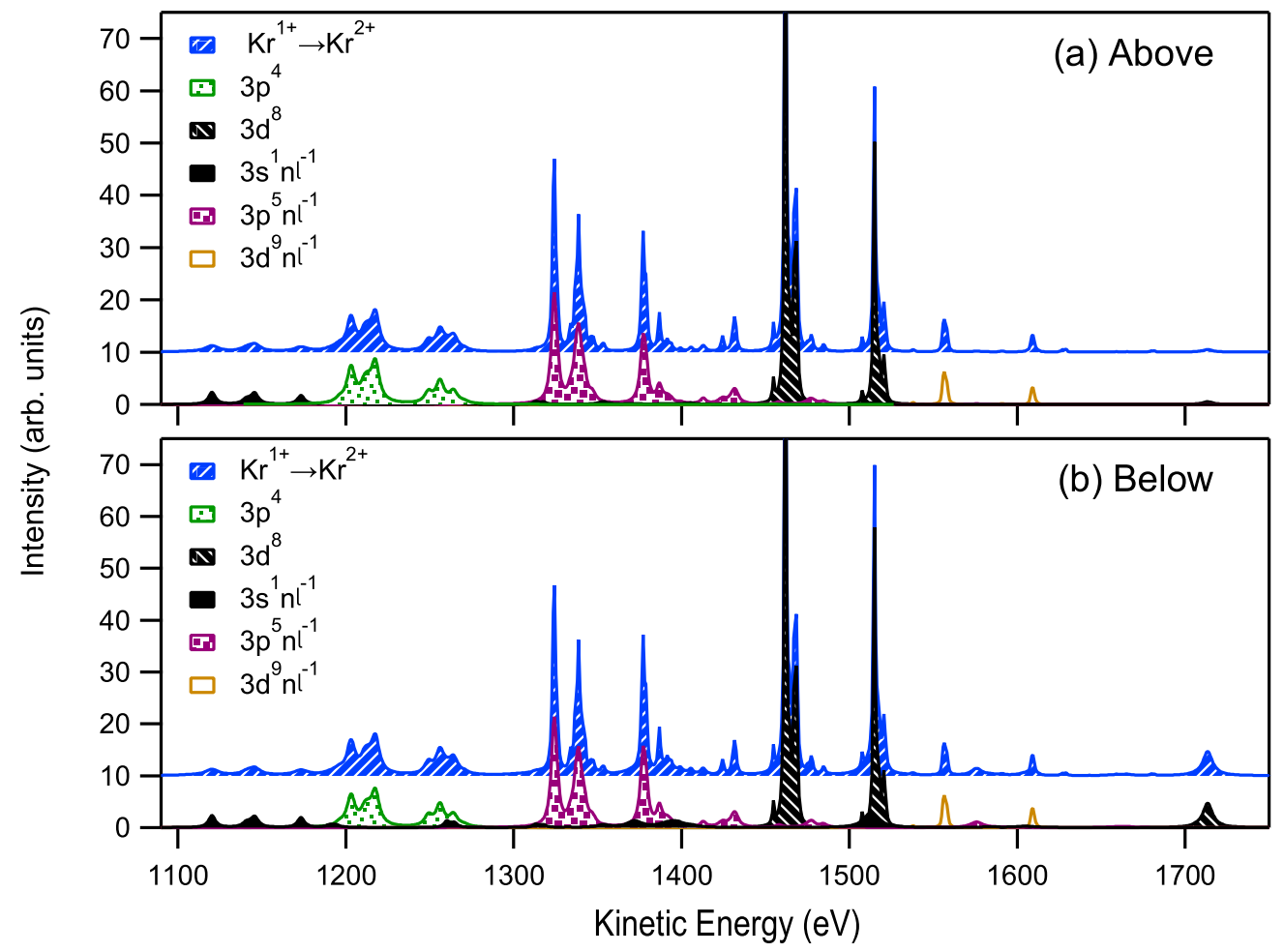

FIG. 3. Partial $\mathrm{Kr}^{1+} \rightarrow \mathrm{Kr}^{2+}$ contribution to total Auger spectrum (a) above and (b) below the 1s ionization threshold. The different colors and patterns in the lower part of each panel indicate the contribution of transitions to individual final-state configurations.

\section{1. $\mathrm{Kr}^{1+} \rightarrow \mathrm{Kr}^{2+}$ Auger transitions}

As mentioned above, $\mathrm{Kr}^{+}$ions with a hole in the $L$ shell can be populated by process (1) and along the first two lines of process (2).

In Fig. 3, we show the calculated partial Auger spectra associated to the $\mathrm{Kr}^{1+} \rightarrow \mathrm{Kr}^{2+}$ transition. As mentioned above, for these spectra, the lifetime broadening contributions of the initial and the final states are taken into account. The transitions are further divided into different final-state components arising from the decay of $(2 s 2 p)^{-1}$ vacancies above [Fig. 3(a)] and below [Fig. 3(b)] the $1 s$ ionization threshold. Despite the different population mechanisms, the two spectra are very similar since the $2 s^{-1}$ hole decays predominantly via Coster-Kronig Auger decay, which does not contribute in this energy region; see below. Note that the Coster-Kronig decay is a special Auger process where one of the two induced vacancies is in the same shell as the initial vacancy. In the case that both induced vacancies are in the same shell as the initial vacancy, the process is called a super Coster-Kronig decay. Coster-Kronig, and in particular super Coster-Kronig, processes exhibit large Auger rates and lead to broad lines. The calculations include all energetically allowed $\mathrm{Kr}^{2+}$ configurations that can be constructed from the ground-state configuration of $\mathrm{Kr}$. The calculations are in good agreement with the experiment. In particular, we predict all the spectral features, although only the major contributions to the peaks are shown in Table II and Fig. 3. The main spectral features arise from filling the holes in the $L$ shell induced by direct photoionization (below threshold) and by $K L$ emission (above threshold) leading to the doubly ionized states $3 p^{4}, 3 d^{8},(3 s 3 p 3 d)^{-1} 4 l^{-1}$, which can be found in the kinetic-energy range of 1126-1744 eV. The $L_{2,3} M M$ structures have been reported by Morishita et al. using a photon energy of $1850 \mathrm{eV}$ [6]. The main colors and patterns of the sum spectra are the same as represented in Fig. 2 in order to simplify comparison between the spectra below and above threshold.

A detailed comparison of the calculated $L M X$ Auger spectra below and above threshold shows subtle variations in the relative intensities. In particular, the spectral feature at $1710 \mathrm{eV}$ is strongly suppressed above threshold. It is assigned to the $L_{1} M X$ transition, whose initial state cannot be populated by $K L$ emission due to dipole selection rules.

The calculation of photoionization cross sections allowed us to support the observed behavior: between the two selected excitation photon energies 13 and $16.5 \mathrm{keV}$, the $L$-shell cross sections decrease by a factor of $\sim 2$. The $1 s$ photoionization cross section at $16.5 \mathrm{keV}$ photon energy is 11 times larger than the $2 s$ cross section, 27 times larger than $2 p_{3 / 2}$, and 48 times larger than $2 p_{1 / 2}$. Consequently, the most intense $L M X$ decays observed above threshold are due to the $K L_{2,3}$ fluorescence decay. In Fig. 3, the main features are similar between above and below threshold. The reason behind is that the most prominent features arise from the decay of $2 p$ hole states, and the $K L_{2} / K L_{3}$ fluorescence decay ratio as well as the $2 p_{1 / 2} / 2 p_{3 / 2}$ photoionization cross-section ratio at $13 \mathrm{keV}$ follow the statistical approximation of $\sim 0.5$.

The differences between the $L$-shell Auger spectra below and above the $1 s$ threshold in $\mathrm{Kr}$ are similar to those which have been observed for Xe by Piancastelli et al. [3]. In Xe, however, the differences are more pronounced than in $\mathrm{Kr}$. The reason for the differences is that $L_{1} L_{2,3} M$ Coster-Kronig decays are intense in $\mathrm{Kr}$, but, due to energy conservation, 


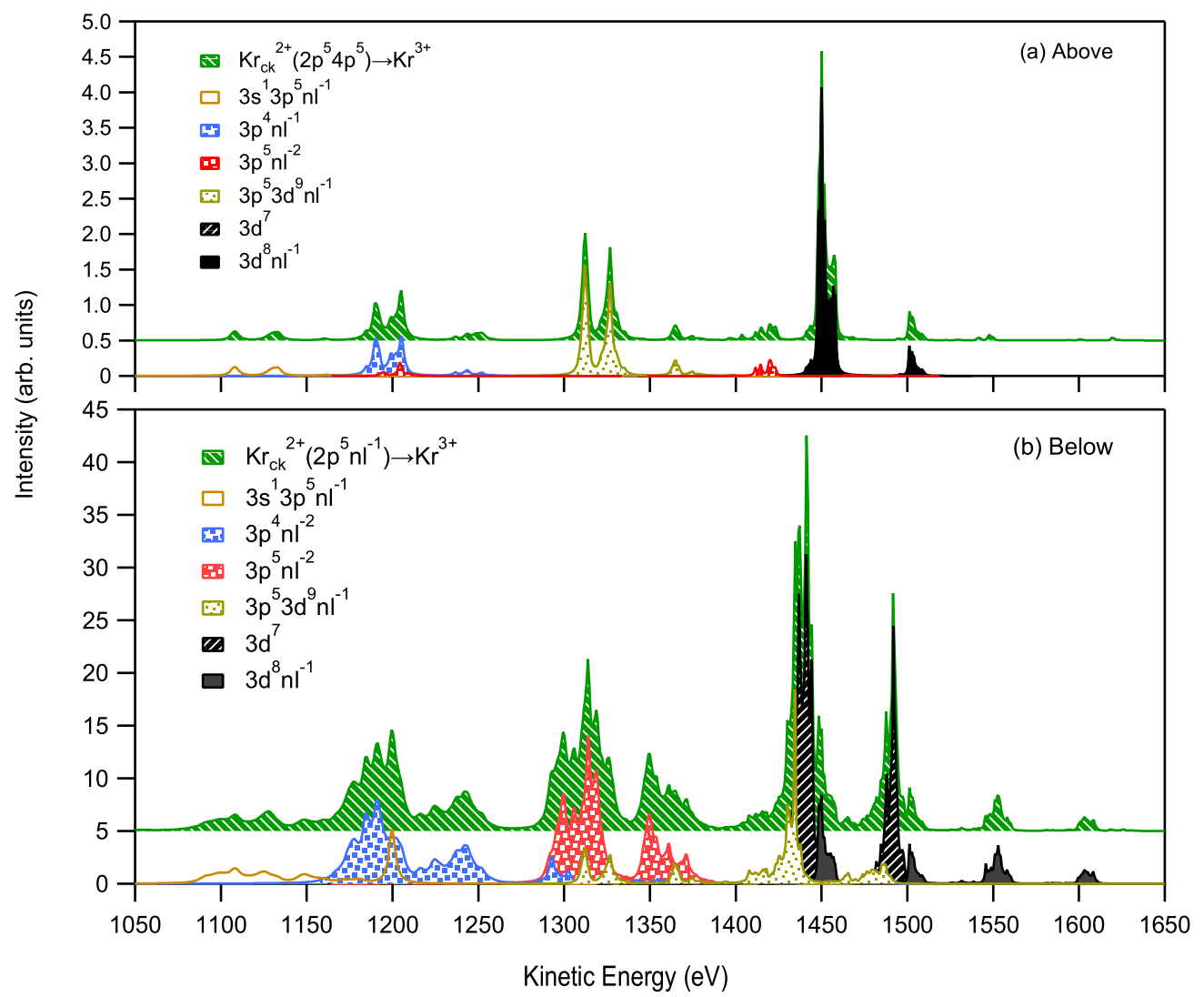

FIG. 4. Partial $\mathrm{Kr}_{C K}^{2+} \rightarrow \mathrm{Kr}^{3+}$ contribution to the Auger spectrum (a) above and (b) below the $1 s$ ionization threshold. The different colors and patterns in the lower part of each panel indicate the contribution of transitions to individual final-state configurations.

forbidden in Xe. This is reflected by the lifetime widths; see, e.g., the ones of Krause and Oliver resulting in $\Gamma\left(L_{1}\right)=4.28$, $\Gamma\left(L_{2}\right)=1.31$, and $\Gamma\left(L_{3}\right)=1.17 \mathrm{eV}$ for $\mathrm{Kr}$, and $\Gamma\left(L_{1}\right)=$ 3.64, $\Gamma\left(L_{2}\right)=3.40$, and $\Gamma\left(L_{3}\right)=3.13 \mathrm{eV}$ for Xe [23]. As a result, the relative strength of $L_{1} M X$ Auger transitions of the singly charged ion is weak in $\mathrm{Kr}$, but strong in $\mathrm{Xe}$.

\section{2. $\mathrm{Kr}^{2+} \rightarrow \mathrm{Kr}^{3+}$ Auger transitions}

Assuming that contribution from direct double photoionization can be neglected, in the present experiment $\mathrm{Kr}^{2+}$ ions with holes in the $L$ shell can be obtained by two processes described in the following.

The first process is Coster-Kronig decay,

$$
\mathrm{Kr}^{1+}\left(L_{1,2}\right) \rightarrow \mathrm{Kr}^{2+}\left(L_{2,3} X\right)+e_{L_{1,2} L_{2,3} X}
$$

where both $2 s^{-1}$ and $2 p_{1 / 2}^{-1}$ initial states are possible from direct photoionization; in addition, $2 p_{1 / 2}^{-1}$ initial states can be populated by $K L_{2}$ x-ray emission. The Coster-Kronig Auger spectra were not measured in the present experiment, but from the calculations it can be noted that the energy range of the $L_{1} L_{2,3} X$ Auger spectrum is at about $4-223 \mathrm{eV}$ and $L_{2} L_{3} X$ at about 5-26 eV.

The second process to induce $\mathrm{Kr}^{2+}$ ions with holes in $L_{1,2,3}$ shells is $K L L$ and $K L X$ Auger decay, i.e.,

$$
\mathrm{Kr}^{1+}(K) \rightarrow \mathrm{Kr}^{2+}(L L)+e_{K L L}
$$

and

$$
\mathrm{Kr}^{1+}(K) \rightarrow \mathrm{Kr}^{2+}(L X)+e_{K L X}
$$

These processes can occur only after $1 s$ ionization. Also, these electrons are not observed directly because, in contrast to the first process, their kinetic energies are well above the presently observed energy region. For example, the calculated kineticenergy range of $K L L$ Auger spectrum is in the range of 10.4$10.9 \mathrm{keV}$. Note that in process $(4),(2 s 2 p)^{-2}$ states are induced. Their sequential decay leads to the observation of $\mathrm{Kr}^{4+}$ final states, as will be discussed below.

Although the initial states are partially the same, we discuss the spectra of Auger decays of $\mathrm{Kr}^{2+}$ states induced by Coster-Kronig decay and $K L L+K L X$ Auger decay separately. For this purpose, the spectra are labeled as $\mathrm{Kr}_{C K}^{2+}$ and $\mathrm{Kr}_{K L}^{2+}$, respectively.

$$
\text { 3. } \mathrm{Kr}_{C K}^{2+} \rightarrow \mathrm{Kr}^{3+} \text { Auger transitions }
$$

In Fig. 4, the partial Auger spectra associated to the transitions of $\mathrm{Kr}_{C K}^{2+} \rightarrow \mathrm{Kr}^{3+}$ measured above [Fig. 4(a)] and below [Fig. 4(b)] the $1 s$ threshold are shown. The spectrum is separated into components indicated by different colors and patterns defined by the final-state electron configuration. The transitions in Fig. 4(b) (below threshold) arise from the decay of $2 p^{5} n l^{-1}$ initial states populated by Coster-Kronig decay subsequent to direct $L_{1}$ and $L_{2}$ photoionization, where $n l$ stands for $3 p, 3 d, 4 s$, and 4p. In Fig. 4(a) (above threshold), the intensities are predominantly due to Coster-Kronig decay 


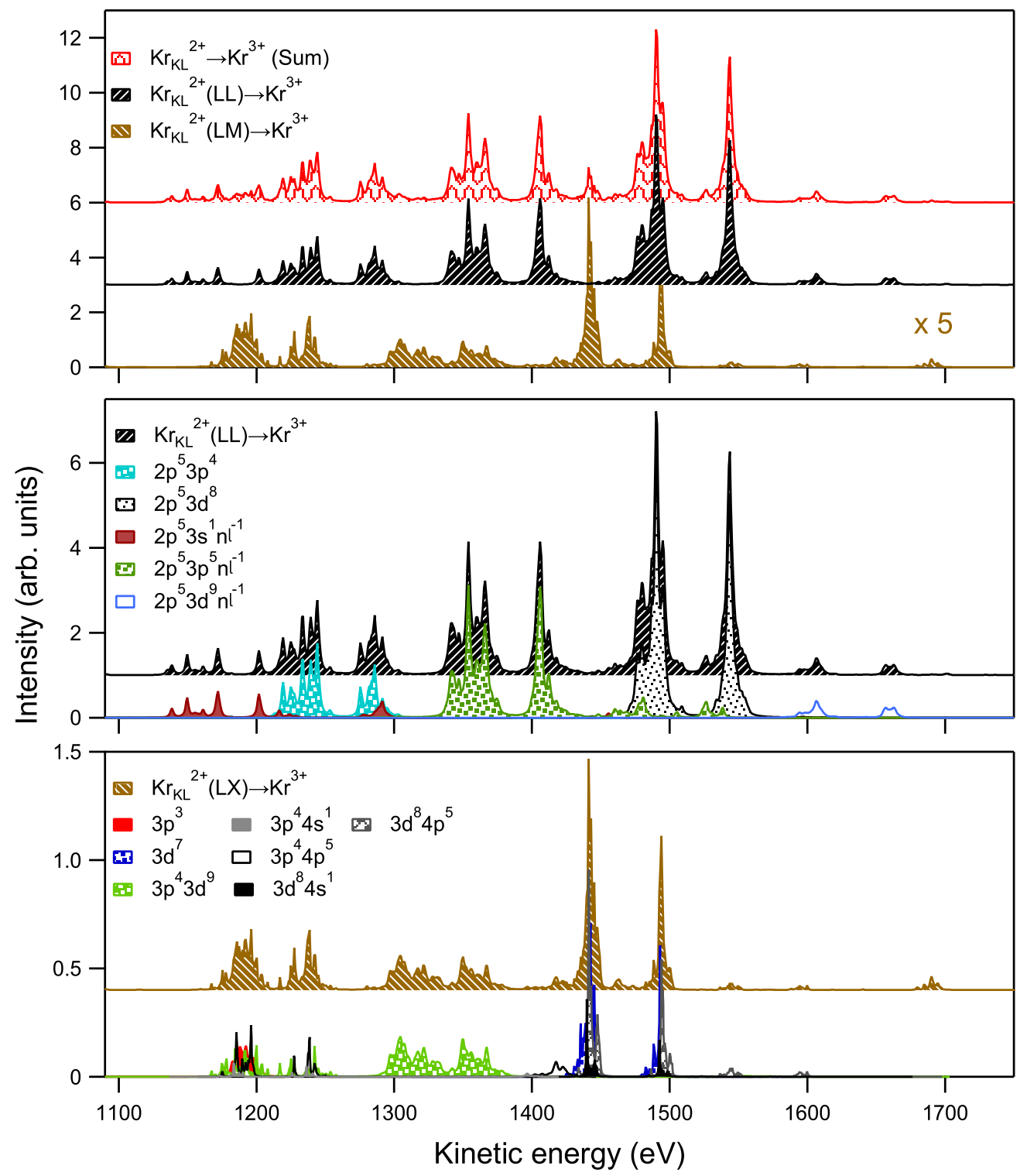

FIG. 5. Partial $\mathrm{Kr}_{K L}^{2+} \rightarrow \mathrm{Kr}^{3+}$ contribution to the total Auger spectrum after $1 s$ ionization. The different colors and patterns in the lower part of each panel indicate the contributions of transitions to individual final-state configurations.

after $\mathrm{x}$-ray fluorescence so that the possible initial states are only $2 p_{3 / 2}^{5} 4 s^{-1}$ and $2 p_{3 / 2}^{5} 4 p^{-1}$.

States $2 p^{5} n l^{-1}$ undergo further Auger relaxation of the type $\mathrm{Kr}_{C K}^{2+} \rightarrow \mathrm{Kr}^{3+}+e_{L X X}$ to triply ionized states, giving rise to final states which lie in the kinetic energy of $1099-1711 \mathrm{eV}$ and are detailed in Table II, where $n l$ represents singly or doubly ionized possible orbitals with $n=3$, 4. In summary, the significant differences in the spectra are due to the reduced number of relevant initial-state configurations above threshold as a result of the $\mathrm{x}$-ray fluorescence.

\section{4. $\mathrm{Kr}_{K L}^{2+} \rightarrow \mathrm{Kr}^{3+}$ Auger transitions}

Figure 5 shows the Auger decay of the $\mathrm{Kr}^{2+}$ ions with the $L$ holes populated by the second process subsequent to ionization, and $K L L$ and $K L X$ Auger transitions as described in Eq. (2). These Auger decays lead to the formation of the
$\mathrm{Kr}^{3+}$ ion according to

$$
\mathrm{Kr}_{K L}^{2+}(L L) \rightarrow \mathrm{Kr}^{3+}(L X X)+e_{L L-L X X}
$$

and

$$
\mathrm{Kr}_{K L}^{2+}(L X) \rightarrow \mathrm{Kr}^{3+}(X X X)+e_{L X-X X X},
$$

respectively.

The $\mathrm{Kr}^{3+}(L X X)$ still possess a hole in the $L$ shell and can decay to $\mathrm{Kr}^{4+}$ by emitting an electron into the energy region under the present investigation. Our calculation reveals that the second-step process leading to the production of $L L-L X X$ and $L M-M X X$ double core-hole hypersatellite structures is more important than in the case of $1 s$ ionization of $\mathrm{Xe}$ [3]. This is due to the fact that in $\mathrm{Kr}$, the $K L L$ and $K L X$ Auger yields are calculated to contribute by $25 \%$, and $8 \%$, respectively, to the decay of the $\mathrm{Kr} 1 s$ hole, in contrast to Xe where the $K L L$ and $K L X$ Augers combine to a decay probability of about $10 \%$ of the Xe $1 s$ hole. 


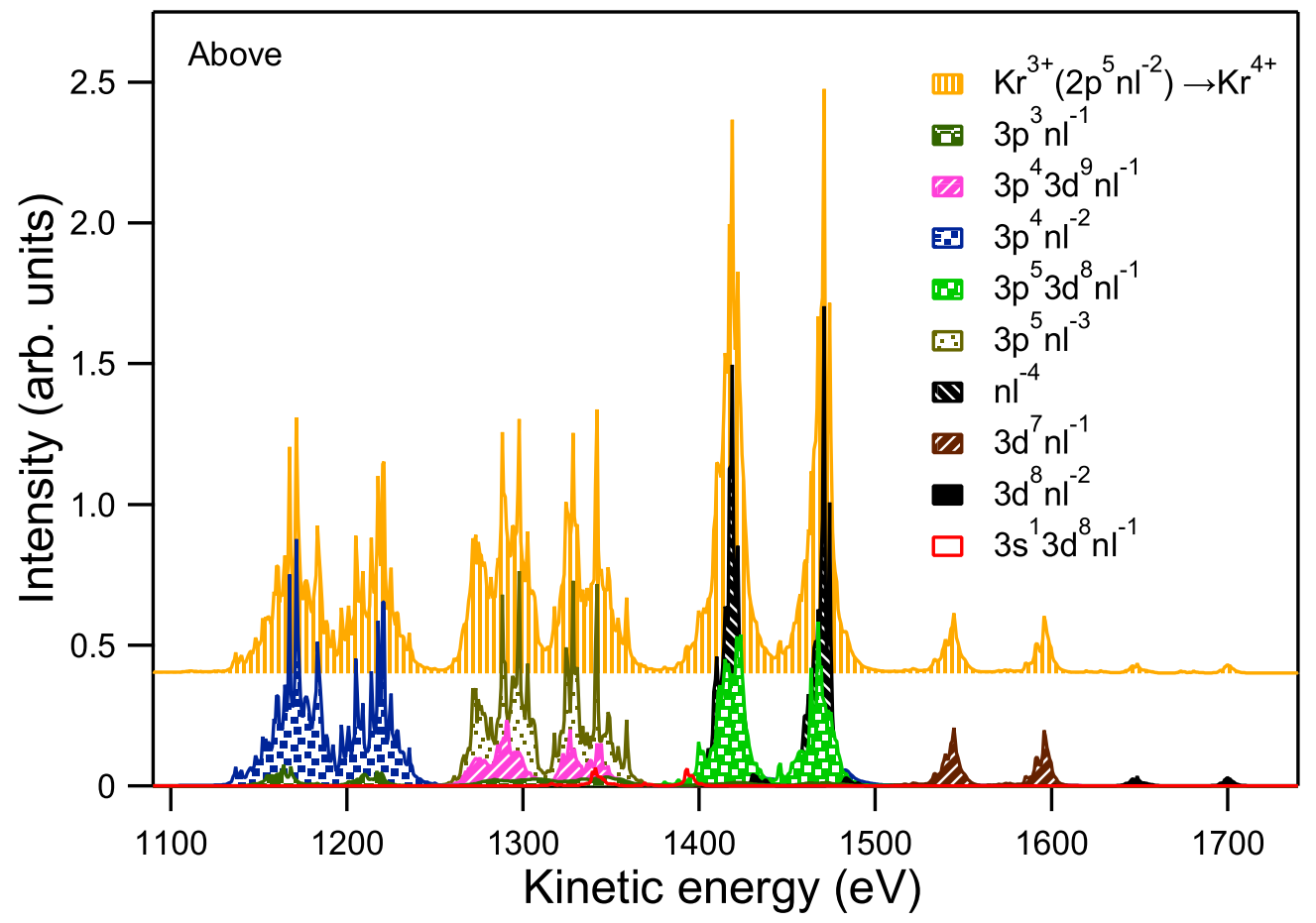

FIG. 6. Partial $\mathrm{Kr}^{3+} \rightarrow \mathrm{Kr}^{4+}$ contribution to the total Auger spectrum after 1s ionization. The different colors and patterns in the lower part indicate the contributions of transitions to individual final-state configurations.

Figure 5 shows the partial Auger spectrum of the $\mathrm{Kr}_{K L}^{2+}(L L) \rightarrow \mathrm{Kr}^{3+}$ transition, associated mainly to the decay of $2 p^{4}$ initial states leading to $2 p^{5} n l^{-2}$ or $2 p^{5} n^{\prime} l^{\prime-1} n l^{-1}$ final states. Here, $n$ and $\mathrm{n}^{\prime}$ are predominantly equal to 3 , i.e., the $L$ hole decays mainly by the $L M M$ Auger.

As stated above for $\mathrm{Kr}$, the $1 s$ core-hole state decays via $K L L$ Auger decay with $25 \%$ probability. To be more specific, $15 \%$ decay to the $2 p^{4}$ configuration, $7 \%$ to $2 s^{1} 2 p^{5}$, and $3 \%$ to $2 s^{0}$. Because of low relative populations of the $2 s^{1} 2 p^{5}$ and $2 s^{0}$ states, their Auger spectra are not shown in Fig. 5. Note also that since the states of the two latter configurations decay predominately via Coster-Kronig processes, their contribution to the $\mathrm{Kr}^{2+}(L L) \rightarrow \mathrm{Kr}^{3+}$ Auger spectrum is significantly smaller than their percentages in populating the $\mathrm{Kr}^{2+}(L L)$ states.

The final-state configurations of these Auger decays are presented in Table II. These structures are seen only above the $K$ edge, such as the structures at kinetic energies of 1490 and $1540 \mathrm{eV}$, which have been assigned by our calculation to be $2 p^{4} \rightarrow 2 p^{5} 3 d^{8}$ transitions. The present spectra are in a good agreement with values predicted with smaller-scale calculations by Oura et al. [1]. For the sake of visibility, the $\mathrm{Kr}_{K L}^{2+}(L M) \rightarrow \mathrm{Kr}^{3+}$ spectrum in Fig. 5 is multiplied by a factor of 5. Its multiplied intensity is comparable to the intensity of the $\mathrm{Kr}_{K L}^{2+}(L L) \rightarrow \mathrm{Kr}^{3+}$ transitions. This can readily be understood by taking into account that the $\mathrm{Kr}_{K L}^{2+}(L L)$ to $\mathrm{Kr}_{K L}^{2+}(L X)$ population ratio is $\cong 3: 1$ and that in the case of $\mathrm{Kr}_{K L}^{2+}(L L)$, two $L$ holes can decay in contrast to $\mathrm{Kr}_{K L}^{2+}(L X)$ with only one hole in the $L$ shell.

Finally, we shall compare the Auger energies. The energies of the $\mathrm{Kr}_{K L}^{2+}(L L) \rightarrow \mathrm{Kr}^{3+}$ are generally larger than those of the $\mathrm{Kr}_{K L}^{2+}(L M) \rightarrow \mathrm{Kr}^{3+}$ Auger transitions. This is probably due to the larger Coulomb repulsion of the two holes in the $L$ shell than for one hole in the $L$ and one in the $X$ shell. This difference is in the case of a $\mathrm{Kr}_{K L}^{2+}(L L)$ initial state that also transferred to the Auger electron.

\section{5. $\mathrm{Kr}^{3+} \rightarrow \mathrm{Kr}^{4+}$ Auger transitions}

As discussed above, the $\mathrm{Kr}^{3+}(L X X)$ ions with a hole in the $L$ shell are induced by the decay of $\mathrm{Kr}^{2+}(L L)$ ions. These triply charged ions can further decay via

$$
\mathrm{Kr}^{3+}(L X X) \rightarrow \mathrm{Kr}^{4+}(X X X X)+e_{L X X-X X X X} .
$$

The partial Auger spectrum associated to $\mathrm{Kr}^{3+} \rightarrow \mathrm{Kr}^{4+}$ transitions is shown in Fig. 6. It corresponds to the decay of triply ionized states $2 p^{5} \mathrm{nt}^{-2}$, where $n t^{-2}$ represents doubly ionized $3 p$ and $3 d$ orbitals. The configurations of the quadruply ionized $\mathrm{Kr}$ final states are summarized in Table II.

It should be mentioned that the $L L$ and $L X$ initial states populated by $K L L$ and $K L X$ Auger can also decay via CosterKronig transitions, leading to even higher charged states. Here the mechanism is

$$
\begin{aligned}
\mathrm{Kr}^{2+}\left(L_{1} L_{1}\right) & \rightarrow \mathrm{Kr}^{3+}\left(L_{1} L_{2,3} X\right)+e \\
& \rightarrow \mathrm{Kr}^{4+}\left(L_{2,3} L_{2,3} X X\right)+e \\
& \rightarrow \mathrm{Kr}^{5+}\left(L_{2,3} X X X X\right)+e .
\end{aligned}
$$

The last states can decay to $\mathrm{Kr}^{6+}$ or even to $\mathrm{Kr}^{7+}$, if an additional $L_{2} \rightarrow L_{3} X$ Coster-Kronig decay occurs. However, since at each decay step the initial population spreads over an increasing amount of states, the spectra gradually smear 
into the background. Therefore, these decays are not discussed further in the present work.

\section{CONCLUSIONS}

A detailed study of deep-core photoionization of $\mathrm{Kr}$ atoms has been presented, obtained by a hard x-ray photoelectron spectroscopy experiment, and supported by relativistic multiconfiguration interaction Dirac-Fock calculations. The core-hole lifetime of the $\mathrm{Kr} 1 s$ orbital vacancy has been extracted; binding energies and relative intensities of the satellite structures have also been assigned. Furthermore, we show a detailed analysis of Auger cascade spectra below and above the $1 s$ ionization threshold, starting from a singly ionized state, produced by both direct ionization and $K L$-emission processes, that undergoes further relaxation by Auger cascade leading to multiply ionized states. The role of the $K L$ emission versus $K L L$ and $K L X$ Auger emission on the formation of $L$-shell vacancies and on the Auger cascade intensities, due to the $K L$ emission selection rule, has been stressed. As for the relaxation processes following the ionization of the $1 s$ orbital, the $K L$ emission yields are determined to be $54 \%$, which outweighs Auger emission yields estimated to $33 \%$. $L X-L X X$ Auger structures have been revealed to be not negligible as in the $X e$ case, and their intensities and energy positions have been assigned as results of the second-step $K L L$ relaxation leading mostly to the formation of $\mathrm{Kr}^{3+}$ and $\mathrm{Kr}^{4+}$ ions. In addition to increasing the knowledge of the decay dynamics of medium-sized core-ionized ions, the work can be extended to molecules, such as, e.g., the $\mathrm{Br} 1 s$ threshold of a number of bromine-containing molecules.

\section{ACKNOWLEDGMENTS}

This project has received funding from the European Union's Horizon 2020 research and innovation programme under the Marie Sklodowska-Curie Grant Agreement No. 713606 and from Research Council for Natural Sciences of the Academy of Finland. Experiments were performed at the BL29XU of SPring-8 with the approval of RIKEN (Proposal No. 20160025). The authors are grateful to the members of the engineering team of the RIKEN SPring-8 Center for their technical assistance.
[1] M. Oura, T. Gejo, K. Nagaya, Y. Kohmura, K. Tamasaku, L. Journel, M. N. Piancastelli, and M. Simon, New J. Phys. 21, 043015 (2019).

[2] J. C. Woicik, Hard X-ray Photoelectron Spectroscopy (HAX$P E S$ ), Springer Series in Surface Science Vol. 59 (Springer, New York, 2016), and references therein.

[3] M. N. Piancastelli, K. Jänkälä, L. Journel, T. Gejo, Y. Kohmura, M. Huttula, M. Simon, and M. Oura, Phys. Rev. A 95, 061402(R) (2017).

[4] N. Boudjemia, K. Jänkälä, T. Gejo, K. Nagaya, K. Tamasaku, M. Huttula, M. N. Piancastelli, M. Simon, and M. Oura, Phys. Chem. Chem. Phys. 21, 5448 (2019).

[5] J. Jauhiainen, A. Kivimäki, S. Aksela, O.-P. Sairanen, and H. Aksela, J. Phys. B: At. Mol. Opt. Phys. 28, 4091 (1995).

[6] Y. Morishita, Y. Tamenori, K. Okada, K. Oyama, K. Yamamoto, K. Tabayashi, T. Ibuki, K. Moribayashi, and I. H. Suzuki, J. Phys. B: At. Mol. Opt. Phys. 39, 1323 (2006).

[7] I. H. Suzuki, A. Fujii, S. Nagaoka, M. Kosugi, K. Okada, T. Ibuki, S. Samori, Y. Tamenori, and H. Ohashi, J. Phys. B: At. Mol. Opt. Phys. 37, 1433 (2004).

[8] E. I. McGuire, Phy. Rev. A 11, 17 (1975).

[9] S. B. Hansen, K. B. Fournier, A. Ya. Faenov, A. I. Magunov, T. A. Pikuz, I. Yu. Skobelev, Y. Fukuda, Y. Akahane, M. Aoyama, N. Inoue, H. Ueda, and K. Yamakawa, Phys. Rev. E 71, 016408 (2005).

[10] M. Drescher, M. Hentschel, R. Kienberger, M. Uiberacker, V. Yakovlev, A. Scrinzi, Th. Westerwalbesloh, U. Kleineberg, U. Heinzmann, and F. Krausz, Nature (London) 419, 803 (2002).

[11] J. F. Seely, C. A. Back, C. Constantin, R. W. Lee, H.-K. Chung, L. T. Hudson, C. I. Szabo, A. Henins, G. E. Holland, R. Atkin, and L. Marlin, J. Quantum Spectrosc. Radiat. Transf. 99, 572 (2006).
[12] B. Rudek, D. Rolles, S.-K. Son, L. Foucar, B. Erk, S. Epp, R. Boll, D. Anielski, C. Bostedt, S. Schorb, R. Coffee, J. Bozek, S. Trippel, T. Marchenko, M. Simon, L. Christensen, S. De, S.-i. Wada, K. Ueda, I. Schlichting, R. Santra, J. Ullrich, and A. Rudenko, Phys. Rev. A 87, 023413 (2013).

[13] L. Young, D. A. Arms, E. M. Dufresne, R. W. Dunford, D. L. Ederer, C. Höhr, E. P. Kanter, B. Krässig, E. C. Landahl, E. R. Peterson, J. Rudati, R. Santra, and S. H. Southworth, Phys. Rev. Lett. 97, 083601 (2006).

[14] B. Krässig, J. C. Bilheux, R. W. Dunford, D. S. Gemmell, S. Hasegawa, E. P. Kanter, S. H. Southworth, L. Young, L. A. LaJohn, and R. H. Pratt, Phys. Rev. A 67, 022707 (2003).

[15] A. Filipponi, D. T. Bowron, C. Lobban, and J. L. Finney, Phys. Rev. Lett. 79, 1293 (1997).

[16] L. O. Werme, T. Bergmark, and K. Siegbahn, J. Physica Scripta 6, 141 (1972).

[17] A. Kovalík, V. M. Gorozhankin, A. F. Novgorodov, A. Minkova, M. A. Mahmoud, and M. Ryšavý, J. Electron Spectrosc. Relat. Phenom. 58, 49 (1992).

[18] K. Tamasaku, Y. Tanaka, M. Yabashi, H. Yamazaki, N. Kawamura, M. Suzuki, and T. Ishikawa, Nucl. Instrum. Methods. A 467-468, 686 (2001).

[19] The analyzer as well as the gas cell were originally supplied by Gammadata Scienta.

[20] L. Asplund, P. Kelfve, B. Blomster, H. Siegbahn, and K. Siegbahn, Phys. Scr. 16, 268 (1977).

[21] R. D. Deslattes, E. G. Kessler, P. Indelicato, L. de Billy, E. Lindroth, and J. Anton, Rev. Mod. Phys. 75, 35 (2003).

[22] J. L. Campbell and Tibor Papp, At. Data Nucl. Data Tables 77, 1 (2001).

[23] M. O. Krause and J. H. Oliver, J. Phys. Chem. Ref. Data 8, 329 (1979). 
[24] D. Céolin, J. Ablett, D. Prieur, T. Moreno, J.-P. Rueff, T. Marchenko, L. Journel, R. Guillemin, B. Pilette, T. Marin et al., At. Data Nucl. Data Tables 190, 188 (2013).

[25] M. F. Gu, Can. J. Phys. 86, 675 (2008).

[26] I. P. Grant, Springer Science \& Business Media. 40 (2007) (unpublished).

[27] J. Niskanen, K. Jänkälä, M. Huttula, and A. Föhlisch, J. Chem. Phys. 146, 144312 (2017).
[28] S. J. Schaphorst, A. F. Kodre, J. Ruscheinski, B. Crasemann, T. Åberg, J. Tulkki, M. H. Chen, Y. Azuma, and G. S. Brown, Phys. Rev. A 47, 1953 (1993).

[29] O. Dragoun, A. Špalek, and F. Wuilleumier, Czech. J. Phys. 54, 833 (2004).

[30] M. Breinig, M. H. Chen, G. E. Ice, F. Parente, B. Crasemann, and G. S. Brown, Phys. Rev. A 22, 520 (1980). 